\title{
Control systems identification in finance and economics
}

\author{
O. Criner \\ Texas Southern University, Houston, Texas U.S.A.
}

\begin{abstract}
The research program that views economies as complex dynamic systems has received significant development over the past several decades and has become known as "Complexity Economics". The amount of information that economies create has grown exponentially. Most markets on formal exchanges have become electronic and they are consolidating into new organizational structures. The data from worldwide economic activity are widely available. However, the methods of analysis and interpretation of these data have not kept pace.

Everyday hundreds of parameters are reported by corporations, governmental and nongovernmental agencies. Corporations make their earnings reports, financial events, and other information available. This flood of information is continuously analyzed in the various media. However, the effect of this influence is subject to varied interpretation.

The research reported here seeks to define a method by which to quantify this influence using several globally-traded financial instruments. The methodology applies data analysis techniques analogous to those used for control of dynamical systems and links the response of trading activity to reports of economic activity. The important issues are (1) identification of the reports that have significant effect on trading activities in specific markets, and (2) determination of the extent, magnitude, and duration of the influence.

We design a computational dynamical systems model from the derivatives of the market prices and use the external economic data as driving functions on the dynamical system. We explore a financial market system model consisting of the derivatives markets in crude oil, stock indices, and currencies.
\end{abstract}

Keywords: dynamical systems, financial events, economic indicators, event trading. 


\section{Introduction}

Dynamical systems applications in financial markets are receiving considerable attention as the information from financial markets and the ability to process that information becomes widely available. Scientific and engineering techniques are being widely used in the analysis of financial markets. Model building is the essence of scientific inquiry. So, it is natural to consider model building for financial systems. Constructing dynamical systems models of financial markets is an "inverse" problem, since there are no fundamental first principles of market finance as there are for physics or engineering. There are no long standing empirically verified equations describing fundamental first principles from which models can be formulated.

Indeed, the traditional theories of finance have come under question as unsatisfactory descriptions of the market processes [1,2]. The rigorousness of the mathematics does not compensate for a theory the assumptions of which are not supported by empirical scientific evidence. Further, grand theories "in the large" should not be attempted, since the processes we are attempting to model are nonlinear, time varying, and, possibly, chaotic. This research is concerned with the transient responses of financial markets to changes in parameters or financial events. Hence, we attempt to model limited but significant processes that may provide insight on the influence of certain events on the behaviour of market systems.

Information from financial and economic activities has become ubiquitous as the global economy expands. Concerns about economic activity, the prices of essential commodities and services, and the wellbeing of national economies are expressed throughout the media and especially on the all-news media outlets. The development of financial and economic web sites has made a great deal of economic and financial data available to individuals worldwide. Websites offering trading and investment services now number in the tens of thousands. Cursory searches on World Wide Web for various permutations on the strings "investment advisors" and commodity trading advisors" produces millions of hits. This suggests that there is an exceptionally large group of people attempting to "make sense" of economic systems, i.e., people are attempting to make models to analyze and explain the phenomena they observe in the marketplace. This has let to a large number of books, tools and procedures for the study of financial time series.

The range of people engaged in this process is very broad and extends from the professionals responsible for very large investment pools to the individual investor and trader with a small IRA or personal account. There is so much activity that the reporting of financial and economic news now is a major industry in itself, which is in stark contrast to the situation forty years ago when the industry was essentially opaque. The opacity may have been due largely to the lack of an efficient and inexpensive financial and economic information infrastructure. A new trading technique, "event trading", has been developed where traders look for specific events to trigger profitable price movement. This fact suggests that these specific events have specific responses in the market and 
these responses have been characterized by the event traders. One result of this research is to formalize the event surveillance process and to integrate it into a model of the market system. Since financial and economic surveillance has become so all encompassing of the major economic systems and large datasets are more available, it is now possible to construct models to forecast economic and financial events more accurately. Not withstanding the view of markets as complex dynamical systems that behave counterintuitively to many stimuli, we assume that complex systems can behave "linearly" in certain instances, also. Such instances have been observed and are presented herein.

This paper examines the transient response and time-wise local effects of announcements of economic information upon the prices of several derivative instruments. Several sources of economic data are available along with the well known price time histories of the Dow Jones Industrials, the S\&P500, the Russell 2000, and the NASDAQ stock indices and their associated futures markets. We choose to examine the effects of economic data that have been rated as very important to the markets by several authorities. Baumohl [3] and Yarmone [4] provide in-depth discussions and importance rankings for many indicators. Briefing .com [5] provides synoptic information and aggregated daily data reporting for many indicators. Several other websites publish current economic data as do the originating institutions of government, private economic research groups, and industry organizations. Trading sites also provide this information in conjunction with the subscriptions for the use of the trading facilities of the site. The website fxcm.com DailyFX Plus combines Thomson IFR's streaming news and market commentary, real-time charting from DailyFX, and live foreign exchange rates from FXCM's Trading workstation platform [7]. This mass of economic information requires extraordinary effort to combine it with the price time histories of trading instruments of interest and to analyze, interpret, and infer some action on the part of an investor or trader. Clearly some computational techniques need to be developed to assist in these analyses. We analyze the design requirements for such systems, also.

We assume that the stock indices and their associated futures are descriptive of the overall marketplace for exchange traded instruments. The currency markets and their associated derivatives markets are another set of indices of the relative position of economies with respect to each other and they respond rapidly to the announcement of economic data and other events. The commodity markets for raw materials that drive these economies are another major indicator of economic activity and crude oil heads the list of important commodities for a number of indices. The extremely high importance of oil in the economies of the world is indicated in the data by the weights given for inclusion in the various commodity indices. In the Rogers International Commodity Index [8], crude oil is $21 \%$ and Brent oil $14 \%$ of the weights of the index of 36 commodities. The next highest weights are for wheat at $7 \%$, corn at $4.75 \%$, Cotton at $4.05 \%$, copper and aluminium at $4 \%$, Gold at $3 \%$ and all of the others are at $3 \%$ or less. Gold is singled out because of its popularity and purported inflation hedge characteristics. 


\section{Dynamical systems model design}

Dynamical systems model construction depends on the existence of a set of candidate models and systems identification is the name of the process of selecting the parameters for the chosen model of the process from the set. We formulate the problem of market tracking on electronic exchanges as a very elaborate example of the fundamental tracking problem in the control theory of dynamical systems. Figure 1 is a simplified diagram of the tracking problem using the Maxwell viscoelastic model. A spring with spring "constant" $\mathrm{k}$ is hung from a rigid support beam above and an inertial mass $\mathrm{m}$ is attached to the spring and constrained by the walls to move only in one direction. The walls have been coated with a sticky substance with viscosity $v$ that resists motion in proportion to the speed of the motion of the mass. The Little Man, our financial operator, is instructed to keep to keep the pointer $p_{o}(t)$ on the mass of the system as close as possible to the pointer $p_{i}(t)$ being observed from outside the system by applying a force $F(t)$ to the mass $m$. Grodins [9] uses the Little Man controller in the study of biological control models, which are, in many ways, very similar to economic and financial control models.

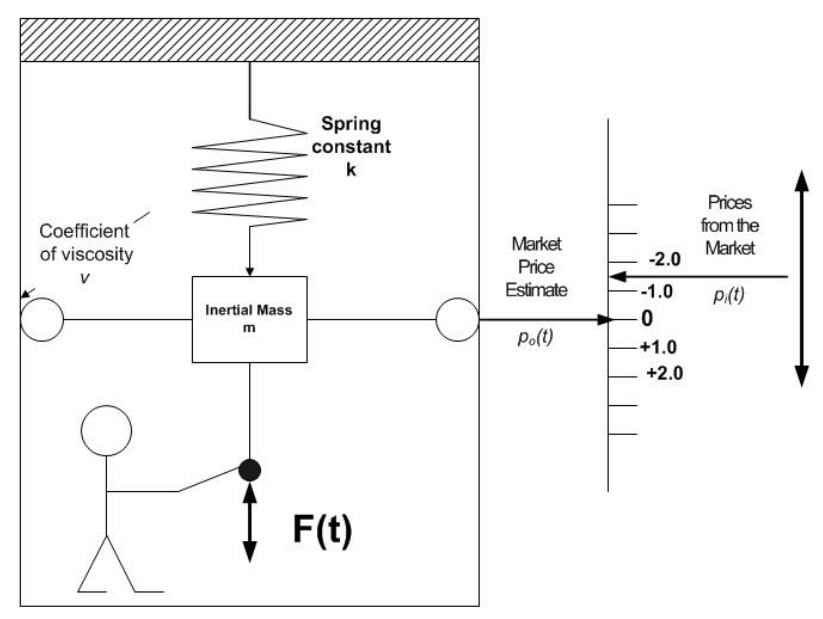

Figure 1: Little Man market tracker.

How is this system to be modeled given, what we know about the incoming data of observed prices? Can the transients, oscillations, and the steady state be identified? In this model the mass, spring "constant", and the viscosity may not be constant at all and may vary in unusual ways. The volume of trading or the open interest in a market may be associated with the mass in the model, in which case it is not a constant. A very thin market will have a small mass and the model will have difficulty tracking the market as the forcing function $F(t)$ attempts to model the volatility. Extremely large volume markets were usually thought of as 
being less volatile and smoother, but with the advent of programmed trading, volatility appears to have increased.

An essential difference between biological dynamical models and economic and financial dynamical models is that, in most cases, the biological system can be studied and modeled in a laboratory. Computational science provides the ability to study phenomena that cannot be studied in a controlled laboratory environment. Models of economic and financial systems are very much like models of the environment and ecological systems, neither of which can be enclosed in a laboratory. However, dynamical models of this kind are well studied and have applications throughout science and engineering. This paper considers a design of how this class of dynamic models may be applied in finance and economics. This simple model can be used as a prototype component for a much more elaborate lumped mass dynamical model of a market.

Many people have observed that some price data have familiar patterns of behavior. Indeed, "technical analysis" is predicated upon pattern recognition; however, the patterns in dynamical systems are different. As an example, one such pattern, a shock took place on October 31, 2007 at around 9:30 a.m. central time, when the U.S. Energy Information Administration (EIA) released its report of crude oil inventories held outside of the strategic oil reserves. Figure 2 shows the real time dynamic price reaction of electronic trading in crude oil futures for delivery in December. The shape of the upper curve is very similar to the response of a linear dynamical system experiencing a step function force. The price movement amplitude before and after the announcement event is small compared to the magnitude of the jump. The announcement occurred at 10:30 a.m. Eastern Time or 9:30 Central Time, where the writer observed the phenomena and took this screen shot. This is a classic trajectory formation in applied mathematics that is widely modeled and used in dynamical systems. The

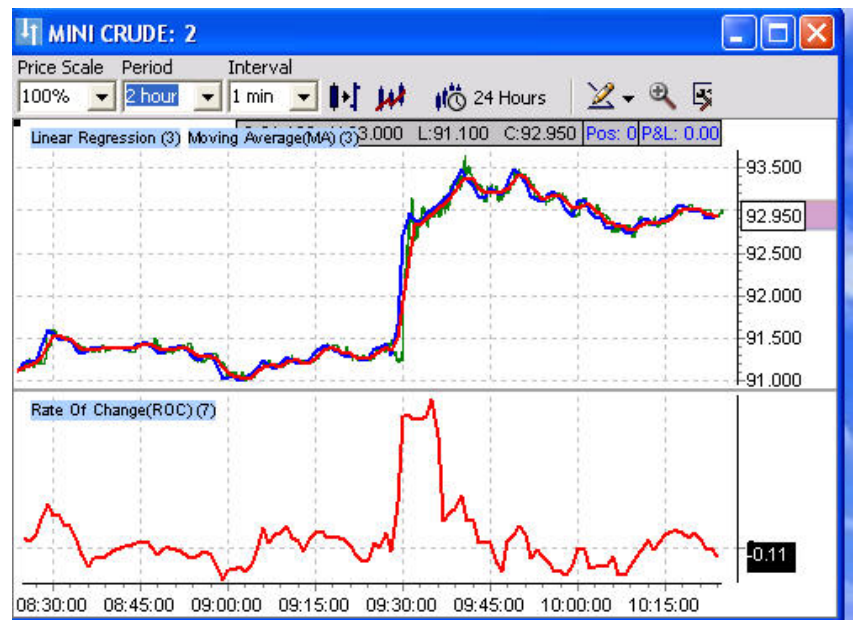

Figure 2: Real-time crude oil futures trading prices. 
lower curve is the dynamic real time rate of change or first mathematical derivative of the upper curve. The rate of change is exactly what is expected of the derivative of a step function, an approximation to the impulse function.

This phenomenon suggests that this event process can be modeled with a linear system over small time intervals. This event may be characterized historically by analyzing the average evoked response of the crude oil market to the change in inventories announcement event.

Piecewise linearization of a nonlinear function is common in applied mathematics as a method for handling nonlinear processes. In cases for which there is almost no fundamental theory one can argue, by analogy with systems having similar responses, what the underlying dynamic process might be, and, therefore, the model for which parameters should be sought. A step function or a sinusoid with a step could be used as a model of the crude oil futures market in the neighborhood of an inventory announcement. These are simple well known functions that could be used to characterize the response of the market, provided that a table of responses as a function of inventory is compiled from history. Such data would constitute the transfer response function of the crude oil market system to the step function announcement event.

Figure 3 shows a closer examination of the event after the fact using two minute historical sampled data. Clearly, some information was lost from the real time data of Figure 2. However, the step function and its derivatives are clear. The mathematical model of this data is shown in Figure 4 as a sine function superimposed on a linear function with a step. The first panel is the actual data and a smoothed estimate of the data. The second panel graph is of the first derivative of the smoothed estimate of the price and the third panel graph is the second derivative. Figure 4 shows the same calculations for the model.
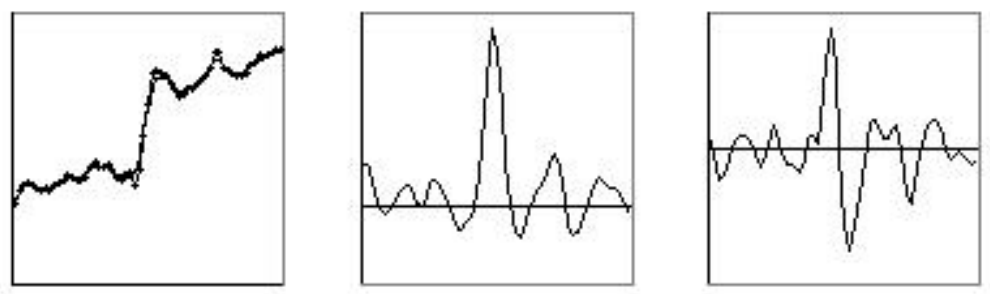

Figure 3: Two minute historical data around the event.

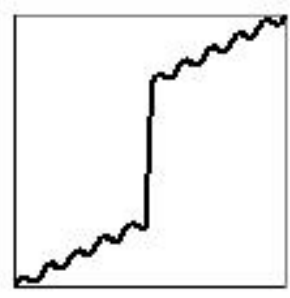

Figure 4:
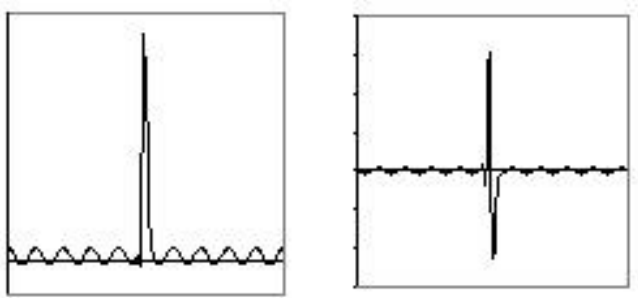

Sinusoid with a step and derivatives. 
Step functions are widely used in applied mathematics and dynamical systems engineering and have well known properties.

\section{Models of the event data}

Since the price of crude oil is the heaviest component of most of the commodity indices, it is important to have an estimator of the futures prices that is based upon the actual market reaction to the economic data from which the change in inventories is derived. The source data are made available in a weekly updated time series of crude oil stocks from the US. Energy Information Administration (EIA). The announcement that is widely reported is of the change in the inventories. The change in inventories, when taken alone, is too volatile to make any reasonable estimates, as most differences of raw data are erratic. However, the actual inventory of oil stocks is a much smoother function. Figure 5 shows a graph of the EIA oil stocks data, a smoothed estimate of the data, $1^{\text {st }}$ and $2^{\text {nd }}$ derivatives of the smoothed estimate, a polynomial model of the smoothed data, and a histogram of the announcement event data. The announcement event data is the difference of the value on one oil stocks inventory report and on the previous report. Since the economic data are sampled at longer intervals and are generally low frequency data, models for these data are easier to construct. They can be constructed automatically using the Microsoft Office Suite. Hence, this capability is widely available.

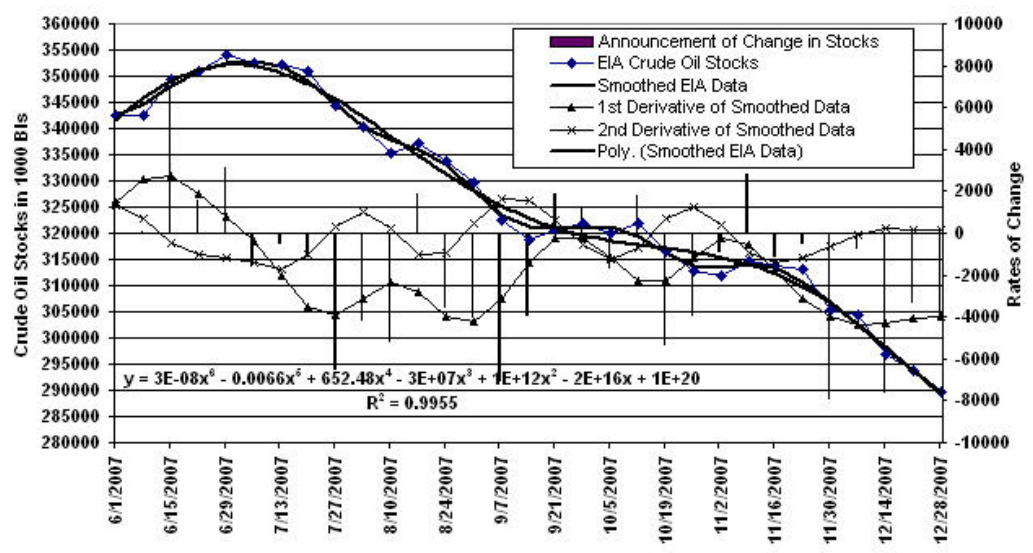

Figure 5: $\quad$ Crude oil stocks and change data.

The smoothed EIA data are used to compute the derivatives. The least squared polynomial is computed on the smoothed data set and its R-squared value is .9955 , which indicates that the smoothed data are very close to the values of the least squared polynomial over that interval. The smoothed data can be reproduced within the interval from the derivatives using a modified predictor-corrector integration scheme and a forecast can also be made. 


\section{Lumped parameter models}

In financial markets we consider the time series of the price-time history of a traded instrument to be its trajectory. So, each instrument has a trajectory or time series that its dynamical system model must track. In addition these tracking dynamical system models are not independent of each other. A more realistic and complex model would be a lattice of mass-spring-dashpot models connected together in order to model the mutual influence of the values of multiple instruments.

Dynamical systems modeling using lumped parameters have been used to study extremely complex applications in mechanics, nanotechnology, and medicine in recent years. There does not appear to be many such operating models in economics and finance, even though there are many papers addressing dynamic systems in economics and finance. We want to study the transient response of several instruments to economic events using a model like that described above.

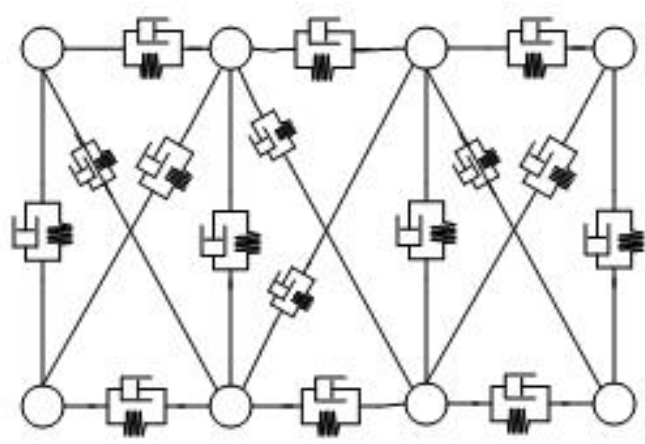

Figure 6: Two dimensional mass-spring-dashpot lattice model.

Figures 7 and 8 shows the trajectories of six related instruments traded on electronic futures markets on 31 October 2007. We consider the time series of prices of the $100 \mathrm{oz}$ Gold, U.S. Dollar Index, Euro Currency, British Pound, Emini Dow Jones Industrial index, and Emini S\&P500 index contracts, respectively. An examination of Figures 7 and 8 show the effect of the crude oil announcement at around 09:30, since the announcements are Eastern Time and the time series are Central Time. However, the FED event produces the strongest reaction in these markets.

The electronic crude oil and the Japanese Yen futures are also considered. Each node will represent one of these instruments. The research will seek to determine the real time transient response that propagates through the markets as a result of events and announcements. The effect of economic events, the reports of economic parameters on interrelated markets, or the relationship between the dominant commodity, crude oil, and the other trading instruments can be modeled in this manner. The scheduled economic events are shown in Figure 9. 

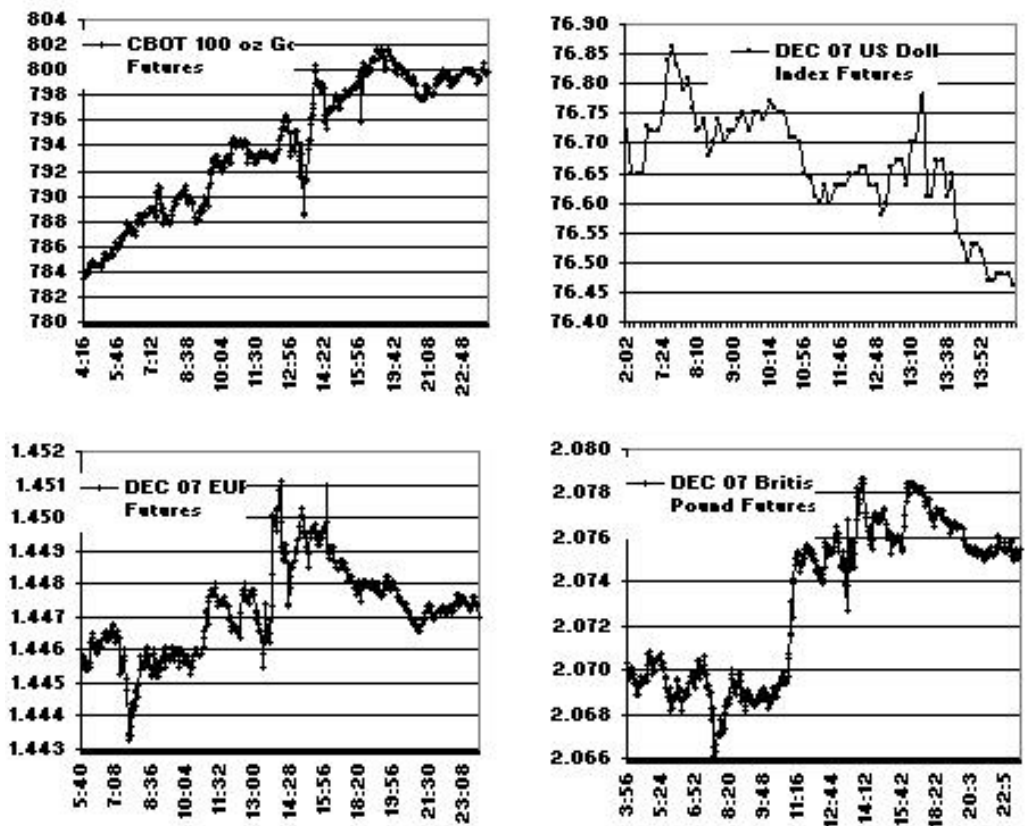

Figure 7: $\quad$ Real time trajectories of 4 related instruments on 31 Oct 2007.
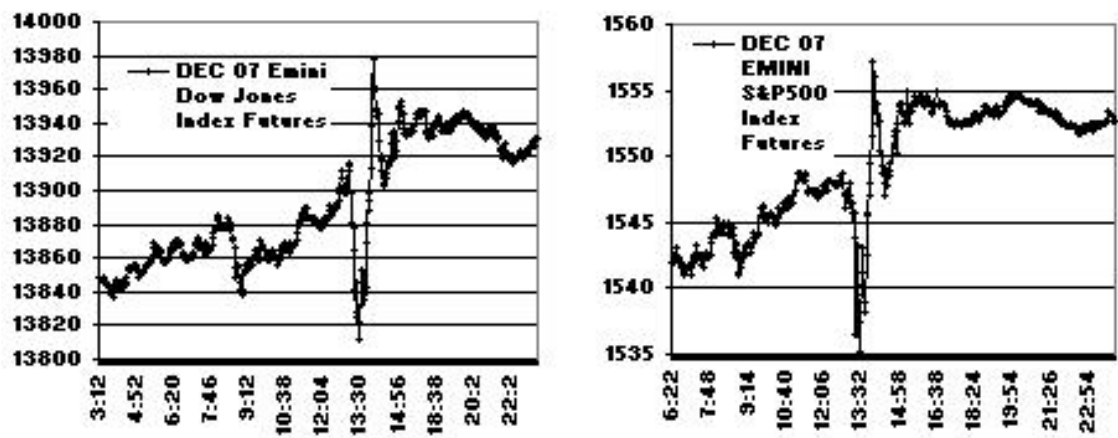

Figure 8: $\quad$ Real time trajectories of 2 related instruments on 31 Oct 2007.

\begin{tabular}{|r|r|l|}
\hline $10 / 31 / 07$ & $8: 30$ & Gross Domestic Product -- Advanced. \\
\hline $10 / 31 / 07$ & $8: 30$ & Chain Deflator-Advanced \\
\hline $10 / 31 / 07$ & $8: 30$ & Employment Cost Index \\
\hline $10 / 31 / 07$ & $9: 45$ & Chicago Purchasing Managers Index \\
\hline $10 / 31 / 07$ & $10: 00$ & Construction Spending \\
\hline $10 / 31 / 07$ & $10: 30$ & Crude Inventories Change \\
\hline $10 / 31 / 07$ & $14: 15$ & FED Open Market Committee policy statement \\
\hline
\end{tabular}

Figure 9: $\quad$ Economic events and announcements on 31 Oct 2007. 


\section{Conclusion}

An observation of the behaviour of the crude oil market in the neighbourhood of a scheduled announcement of a monitoring event showed a pattern that is well known in the study of linear time invariant dynamical systems. The contemporary assumption of markets as nonlinear time varying complex dynamical systems precludes such behaviour, in general. However, one of the characteristics of complex systems listed by Forrester [10] is that complex systems behave counterintuitively. What could be more illustrative of counterintuitive behaviour of a complex system than for it to behave as if it were linear? One of the complex behaviours of such systems is that it may be linear at certain times or under certain conditions. This means that the dynamical system models must also be capable of similar behaviour.

This study of the behaviour of markets in the neighbourhood of significant scheduled events showed that they entered into a quiescent period before the announcement. During that period the behaviour could be modelled as a linear system in the way that mechanics is linear for small deformations. After the event, they behave as struck by impulse functions or step functions. The impulse and step responses decay with time. These observations suggest that nonlinear dynamical system modelling using arrays of masses, springs, and dashpots in lumped models would be a fruitful research program for modelling markets in real time.

\section{References}

[1] James R. Thompson and Edward E. Williams, "Future Enrons await, unless business schools must stop teaching faulty formulas," The Houston Chronicle, Outlook Section, May 27, 2006.

[2] Eric D. Beinhocker, The Origin of Wealth - Evolution, Complexity, and the Radical Remaking of Economics, Cambridge, MA: Harvard Business School Press. 2006.

[3] Baumohl, Bernard, The Secrets of Economic Indicators - Hidden Clues to Future Economic Trends and Investment Opportunities, Upper Saddle River, NJ: Wharton School Publishing, 2007.

[4] Richard Yarmone, The Traders Guide to Key Economic Indicators, Princeton, NJ: Bloomberg Press, 2004.

[5] Briefing.Com, Economic Calendars, $<$ http://www.briefing.com/Investor

[6] /Public/Calendars/ EconomicCalendar.htm>, Accessed November 30, 2007.

[7] FXCM Web Site for FOREX Capital Markets, www.fxcm.com/dailyfxplus.jsp

[8] Rogers, Jim, Hot Commodities - How Anyone can Invest Profitable in the World's Best Market, New York: Random House Trade Paperbacks, 2007.

[9] Fred S. Grodins, Control Theory and Biological Systems, New York, NY: Columbia University Press, 1963.

[10] Jay Forrester, Urban Dynamics, Cambridge, MA: The M.I.T. Press, Massachusetts Institute of Technology, 1969, p. 109. 